\title{
Procédé de mesure de cavitation à partir des coefficients de débit
}

\section{Process for measuring cavitation using flow coefficient}

\author{
D. Peyrot \\ C.E.T.I.M., Nantes
}

L'écoulement à travers un organe placé sur une conduite entraîne une perte de charge fonction du débit. Lorsque, en un point de l'écoulement, la pression statique absolue atteint la valeur de la tension de vapeur, il apparaît des phénomènes de cavitation qui perturbent l'écoulement et tendent à faire diminuer le coefficient de débit de l'appareil.

Au préalable, il apparait intéressant de rappeler la méthode pour mesurer la perte de charge d'un appareil et comment celle-ci se traduit par des coefficients.

La perte de charge d'un appareil inséré sur une conduite est déterminée par la mesure de la différence de pression entre l'amont et l'aval. Les prises de pression sont implantées dans des sections suffisamment éloignées pour que l'on puisse considérer les veines comme homogènes à ces niveaux (prises situées à $5 \mathrm{D}$ à l'amont et $10 \mathrm{D}$ à l'aval). La perte de charge des $15 \mathrm{D}$ de conduite est soustraite de la précédente mesure pour définir celle de l'appareil : $\Delta P$.

Cette perte est fonction du débit $\left(\Delta P=K Q^{2}\right)$, ce qui peut être traduit par un coefficient sans dimension :

$$
\alpha=4 Q \omega^{1 / 2}\left[\pi D^{2}(2 g \Delta P)^{1 / 2}\right]^{-1}
$$

ou sous la forme d'un coefficient :

$C_{v}=1,16 Q(\rho / \Delta P)^{1 / 2}$

avec

- $Q$ débit volumique exprimé en $\mathrm{m}^{3} / \mathrm{h}$

$-\rho$ masse volumique ( 1 pour l'eau)

$-\Delta P$ perte de charge exprimée en bar

$C_{v}=4,6410^{4} D^{2} \alpha$ avec $D$ exprimé en mètres

Le long d'une "ligne de courant", la pression statique absolue évolue, notamment au niveau de la section réglante où il $\mathrm{y}$ a une mise en vitesse importante, elle chute dans une grande proportion pour croitre à l'aval où la section est plus importante. La pression peut atteindre localement la pression de vapeur et entraîner la vaporisation du fluide. A l'aval de la section réglante, la pression peut remonter au-dessus de la pression de vapeur, ce qui entraine la disparition des bulles de gaz induites par la vaporisation. Le niveau de pression à l'aval de l'appareil ne peut pas donner d'indication directe sur les risques de cavitation.

On peut donc avoir pour un même débit selon le niveau général de pression trois états :

- un état non cavitant ou en tout point de la veine fluide, la pression est supérieure à la pression de vapeur ;

- un état de grande cavitation, cavitation en masse ou à partir de la section réglante tout point est à une pression inférieure à la pression de vapeur ;

- un état intermédiaire où la récupération de pression fait disparaitre les bulles précitées à l'aval de l'appareil. (fig. 1) écoulements correspondant aux débits respectifs $Q_{1}, Q_{4}$ et $Q_{3}$.

Le but des coefficients de cavitation est de définir les conditions d'écoulement critique pour lesquelles le robinet risque de caviter.

En réalité, les phénomènes de cavitation dans un robinet n'apparaissent pas brutalement. La pression amont étant maintenue constante, le débit traversant l'appareil étant progressivement augmenté, il apparait que toutes les lignes de courant n'atteindront pas en même temps la pression de vapeur du fluide. Il existe toujours des points de survitesse pour lesquels la pression statique diminue plus rapidement et ou la cavitation se propage en premier, d'où en général une apparition très progressive de la cavitation.

Il devient alors nécessaire de bien définir quel degré de cavitation est adopté pour la définition du coefficient de cavitation.

Les coefficients de cavitation sont donnés par les formules :

$$
C_{f}=\left[\Delta P /\left(P_{1 a}-P_{v}\right)\right]^{1 / 2} \text { ou } K_{f}=\Delta P /\left(P_{1 a}-P_{v}\right)
$$

$\left(\Delta P, P_{1 a}\right.$, et $P_{v}$ étant des pressions exprimées dans une même unité) 

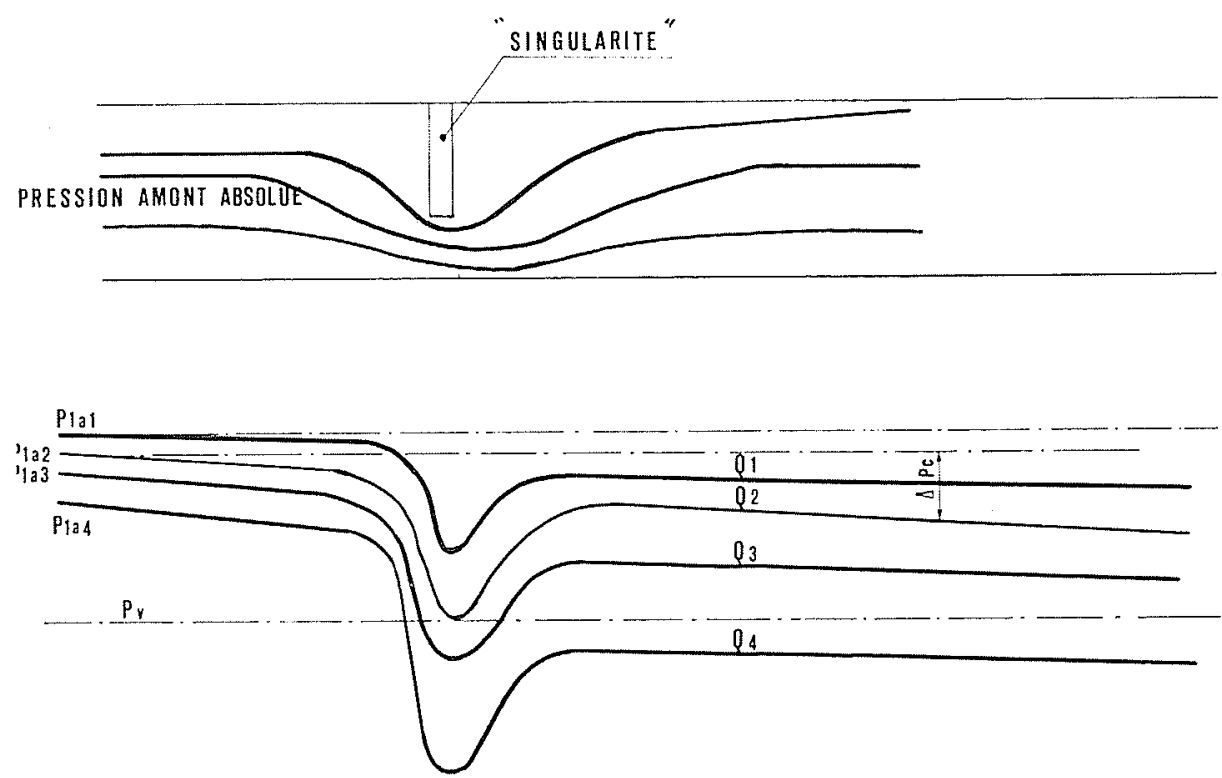

Figure 1. - Lignes de courant au niveau d'une singularité Incidence du débit sur la pression statique le long d'une "ligne de courant".

Pour une ouverture déterminée d'un robinet, il est possible de tracer une courbe $\Delta P=f(Q)$. Si la pression amont est suffisante et qu'à aucun endroit de l'écoulement les lignes statiques n'atteignent la pression de vapeur $P_{v}$, la loi $\Delta P=f(Q)$ est parabolique courbe 1 . (fig. 2). Lorsque la pression amont $P_{1 a}$ diminue, la courbe s'écarte de la parabole au point $M$ et atteint à la limite une saturation, point $N$ de la courbe 2 .

L'évolution du coefficient $\alpha$ se traduit par la courbe 3 .

Si le coefficient de cavitation est choisi au point $M$ dont la détermination est délicate, le critère semble être beaucoup trop sévère. A ce point, les perturbations de l'écoulement sont naissantes et le robinet peut fonctionner sans risque de dégradations à son niveau ou au niveau de la tuyauterie et des organes placés à l'aval.

Si le coefficient de cavitation est choisi au point $N$, le critère n'est pas assez sévère. A ce point de fonctionnement, l'écoulement est fortement perturbé à l'intérieur du robinet et à l'aval, ce qui peut entrainer des détériorations rapides de l'appareil, voire de l'installation. En outre, au niveau de l'expérimentation, la saturation du débil est souvent difficile à alteindre surtout dans les grandes ouvertures.

Pour toutes ces raisons, il semble commode de choisir un critère intermédiaire qui pourrait correspondre à une chute du coefficient de débit d'un pourcentage donné.

Ceci conduit à définir trois coefficients de cavitation :

$C_{f c}$ ou $K_{f c}$ pour la cavitation naissante

$C_{f s}$ ou $K_{f s}$ pour la valeur correspondant au débit de saturation

$C_{f i(3)}$ ou $K_{f i}(3)$ pour la cavitation industrielle, chute du coefficient de débit $\alpha$ (ou $C_{v}$ ) de $3 \%$.

Ce critère peut être modulé au niveau du pourcentage en fonction des types d'appareils ou plus exactement en fonction des coefficients de débit.

Le coefficient de cavitation ainsi présenté se rapproche de celui couramment utilisé dans les turbomachines pour la détermination du NPSH.

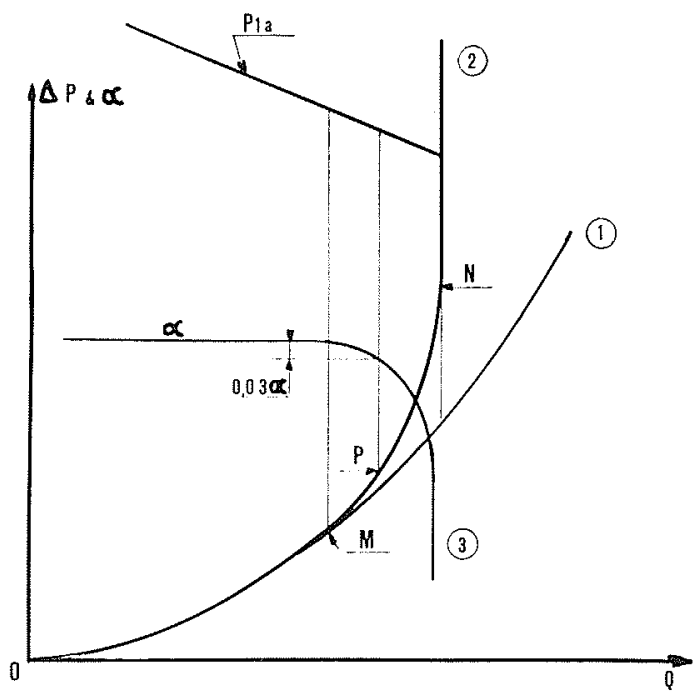

Figure 2. - Critères de cavitation en laisison avec la perte de charge en fonction du débit.

Il a l'avantage d'être facilement déterminé; son exploitation par le fabricant lui permet de déterminer les limites raisonnables d'utilisation de son matériel.

\section{Remarques}

D'autres critères de cavitation peuvent être choisis : détection des premières bulles ou de figures de cavitation, ces méthodes requièrent du matériel plus sophistiqué (visualisation, capteur de fluctuations de pression type hydrophone), elles peuvent être employées en laboratoire pour l'essai d'un prototype. Elles semblent difficilement utilisables pour des essais industriels ceci d'autant plus que leur interprétation est bien des fois délicate. (Notamment, l'apparition des premières fluctuations s'effectue bien avant la dégradation des caractéristiques, ce qui met en évidence la difficulté d'apprécier la position du point $M$ ). 